\title{
A high fidelity cost efficient tensorial method based on combined POD-HOSVD reduced order model of flow field
}

\author{
Mohammad Kazem Moayyedi ${ }^{\mathrm{a}}$ and Milad Najaf beygi ${ }^{\mathrm{b}}$ \\ aCFD and Turbulence Research Laboratory, Department of Mechanical Engineering, University of \\ Qom, Qom, Iran; 'Department of Mechanical and Aerospace Engineering, The University of \\ Oklahoma, Norman OK, USA
}

\begin{abstract}
Computation time and data storage is a significant challenge in every calculation process. Increasing computational speed by upgrading hardware and the introduction of new software are some of the techniques to overcome this challenge. One of the most interesting methods for fast computations is the reduced order frameworks. In this study, the aerodynamic coefficients of the NACA0012 airfoil in subsonic and supersonic flows have been reconstructed and estimated by a cost-efficient form of combined proper orthogonal decomposition-high-order singular value decomposition (POD-HOSVD) scheme. The initial data ensemble contains some members related to the variations of the angle of attack and Mach number. To reduce the computation time, the structure of the standard combined POD-HOSVD approach has been changed to a cost-efficient format. The present method is a grid independent formulation of standard combined POD-HOSVD for the fields with a large number of elements and several effective variables. Results indicate more than 90 percent reduction in the calculation time compares with computational fluid dynamics and standard combined POD-HOSVD methods for a subsonic flow field.
\end{abstract}

\section{ARTICLE HISTORY}

Received 28 August 2018

Accepted 19 November 2018

\section{KEYWORDS}

Proper orthogonal decomposition; HOSVD; compressible flow; reduced order model; fast data estimation

\section{Introduction}

Today engineers are challenging with time-consuming numerical solutions and introducing new software programs, and optimal methods are the primary goal of them. So reduce order methods have a significant impact through this path. The most optimal reduce order model used for the first time by Karhunen and Lumley in 1946 at the same year but separately, was named proper orthogonal decomposition (POD), which is also known as an extension of (K-H) (Karhunen, 1946; Lumley, 1967). Later, this method was applied to various fields. Up to 1980, proper orthogonal decomposition method has been used often in statistical big data analysis. Nowadays, this approach is applying to a variety of issues such as image processing, data 
compression, signal analysis and the identification process. As a reduced order model, the benefits of POD functions in comparison with the other modes are as followed.

POD systems are often nonlinear equations. The POD modes derived simply by the output data of numerical simulations, but in other methods, equations must be linearised to consider on a case as an answer. Also in other methods, the calculation of eigenvalues and eigenvectors produce huge data matrices, which increase the calculation volume and make some difficulties in the computational process. In the other side, POD functions are optimal. So, there are no other modes that can express field in a lower order than POD functions.

Recent progress in POD application, at turbulent flow problem, could reduce a large number of numerical calculations. Modelling the turbulent flow problems by POD had been started since 1967. Lumley used this method for the first time to model the structure of nonhomogeneous turbulence in atmospheric turbulence flow and radio wave propagation. However, a significant development in the proper orthogonal decomposition method occurred when the index technique was used in the context of this method in 1987 by Sirovich and Kirby (1987). He used the index technique to create a process in the determination of human's face. With this development, it was possible to apply any non-square matrix to provide initial data for reducedorder models based on proper orthogonal decomposition. Later, KarhunenLoeve expansion was used as a powerful method in dynamical systems, reduced order modelling of the unsteady flow, turbulence flow and its interaction with structure (Chambes, Adrian, Moin, Stewart, \& Sung, 1988; Dowell et al., 1999; Holmes, Lumley, \& Berkooz, 1996; Romanousk, 1996). Estimation of the shock wave location in high-speed flow is another noteworthy application of POD (Lucia, 2001). Bui-Thanh et al. (2004) used proper orthogonal decomposition to study aerodynamic data reconstruction and inverse design. He mixed experimental and numerical data to provide initial data or snapshots matrix for the POD process. His case of the study was an incomplete data set of compressible external flow. He successfully applied POD on inverse design, also determined the lost data in the initial database (Bui-Thanh, Damodaran, \& Willcox, 2004). POD was also used in database generation of F16 aeroelasticity in various Mach numbers and angle of attacks. Modelling the turbulent velocity structures of an open problem channel flow and reduce order modelling of the supersonic developed compressible and transonic flow around an airfoil for studying the small deformations of the airfoil is also part of the applications of this approach (Bergmann \& Cordier, 2008; Bourguet, Braza, \& Dervieux, 2011; Connell, Kulasiri, Lennon, \& Hill, 2007; 1993; Leschziner, Fishpool, \& Lardeau, 2009; Lieu \& Farhat, 2005; Moayyedi, Sabour, Najafbeygi, \& Hojaji, 2013; Sharma, 2009). 
The tensorial express of POD needs high-order singular value decomposition (HOSVD) as a method to decompose a tensor to some matrices (Lathauwer, Moor, \& Vandewalle, 2000). This method has been applied for classification of handwriting by Berkant et al. (2006). HOSVD was used for modelling the turbulence flow around NACA0012 2D and 3D models to Study the dynamic stall by Martinat et al. (2008) (Berkant and Lars, 2006; Martin \& Senior, 2008). Lorente et al. found the HOSVD as an efficient method to generate an aerodynamic database for a 2D airfoil with the variations of three parameters: Mach number, flap deflection angle and the angle of attack. Authors have achieved the pressure distribution around the surface of the airfoil by HOSVD method (Lorente, Vega, \& Velazquez, 2008). Moayyedi et al. used a combined form of POD and HOSVD model in free vibration simulation of a linear beam under variations of several parameters (Moayyedi, Najafi, \& Najafbeygi, 2014; Najafbeygi, Sabour, Moayyedi, \& Hojaji, 2014). Razvan Stefanescu and his colleagues had research on tensorial proper orthogonal decomposition and compared the results with numerical experiments, standard POD and POD/ discrete empirical interpolation method (DEIM), in a two-dimensional shallow water equation test case. Authors employed a polynomial nonlinearities structure in POD technique to remove the dependence on the dimension of the discretised system. Results show the tensorial POD can reduce the computational cost by up to $76 \times$ times. However, the method was two to eight times slower than the POD/DEIM model. Finally, they used their tensorial method to increases the calculation speed in the off-line stage of POD/DEIM model (Ştefănescu, Sandu, \& Navon, 2014).

Standard combined POD-HOSVD formulation requires a considerable amount of data storage and extensive calculation time for reconstructing flow fields with a large number of elements. But this method can change to a more efficient and less time-consuming approach. The objective of this paper is introducing a method to fast estimate the data from several parameter variations in flow fields with a large number of elements based on cost-efficient combined POD-HOSVD reduced order model. For recovering lost data during the process of numerical simulation, the proper orthogonal decomposition separately was combined with cubic interpolation and cost-efficient HOSVD. The presented method is a powerful approach in the reconstruction of the flow fields with a large number of grids. Calculation time and occupied storage of cost-efficient POD-HOSVD, combined POD-HOSVD and computational fluid dynamics (CFD) methods have been compared. The relative error of each grid, the pressure distribution lines, pressure coefficient and fraction coefficient diagrams on the surface of the airfoil has been compared with the results of the numerical solution. The results indicate the high computational speed and the accuracy of the proposed method. 


\section{Proper orthogonal decomposition}

The expressions of mathematical formulation here closely follow the reference (Bui-Thanh et al., 2004). If consider $\theta(x, t)$ as a random field on a domain $\Omega$. This field can decompose into $\mu(x)$ and time-varying parts $u(x, t)$.

$$
\theta(x, t)=u(x, t)+\mu(x)
$$

At time $t^{n}$, the system displays a snapshot $u_{n}(x)=u\left(x, t^{n}\right)$. The objective in POD is obtaining the most effective characteristic structure $\varphi(x)$ of a set of snapshots of the field $u(x, t)$. Based on the Karhunen-Loeve expansion, Lumley expressed that for coherent structures of turbulent flows, this is equivalent to calculate the basis function $\varphi(x)$. This function maximised the following expression:

$$
\frac{\left\langle(u(x, t), \varphi(x))^{2}\right\rangle}{\langle\varphi(x), \varphi(x)\rangle} \quad \text { With }\|\varphi\|^{2}=1,
$$

which $(f, g)$ express the inner-product in $L^{2}$ space as $\int_{\Omega} f(x) g(x) d \Omega$ and $\|\cdot\|=(\cdot, \cdot)^{(1 / 2)}$ : denotes the norm. Expression (2) means that the projection of field $u$ along $\varphi$ makes a content which is greater than a projection of $u$ along any other basis function. $\langle\cdot\rangle$, denotes the ensemble averaging that can replace by time averaging with the assumption of ergodic flow:

$$
\langle\cdot\rangle=\lim _{T \rightarrow \infty} \frac{1}{T} \int_{0}^{T} d T
$$

Similar to the Gram-Schmidt orthogonalisation procedure, in a subspace that is orthogonal on $\varphi(x)$, maximisation of Equation (2) can be repeated, and this repetition prepares the set of orthogonal functions that defined as POD modes. By using POD modes, $u(x, t)$ can be reconstructed as follow (Bui-Thanh et al., 2004):

$$
u(x, t)=\sum_{k=1}^{N} a^{k}(t) \varphi^{k}(\vec{x}),
$$

where the coefficient $a^{k}(t)$ is uncorrelated, i.e. and is determined by $a^{k}(t)=\left(u(x, t), \varphi^{k}(x)\right)$. To use the POD snapshot, a set of fluctuations, $u_{n}(x)$, with $N$ members in different time steps, $t^{n}$, has been considered as the initial data. The discretisation can be on any other variables which are obtained from CFD simulations or experimental tests.

$$
u_{n}(x)=u\left(x, t^{n}\right)
$$


where $u_{n}$ is unknown at the given time $t^{n}$ in each point of the computational grid. $t^{n}$ is a variable which belongs to parameters such as time, Mach number or angle of attack. Mostly the step size of the $t^{n}$ is considered as a constant amount. Also, from the mathematical viewpoint, the snapshots must be liner independent.

$$
C=\frac{1}{n} \sum_{i=0}^{n-1} u_{i} u_{i}^{T}
$$

where $C$ is the correlation matrix

$$
C \varphi^{k}=\Lambda \varphi^{k}
$$

$\Lambda_{0} \geq \Lambda_{1} \geq \Lambda_{2} \geq \cdots \geq \Lambda_{n-1} \geq 0, k=0,1,2, \ldots, n-1$

where $\varphi$ is the eigenvector, $\Lambda$ is an eigenvalue of the POD and each eigenvector is related to a specify eigenvalue. The POD modes actually can be considered as the spatial correlation matrix eigenfunctions. Hence, POD basis vectors are determined by Equation (5) as eigenvectors of $C$, while the eigenvalues of $C$ are determined with respect to the value of each vector. To determine the eigenfunctions, Sirovich introduced snapshot method that does not need explicitly to calculate the correlation matrix (Bui-Thanh et al., 2004).

If $\theta(\mathrm{x}, \mathrm{t})$ is written as a matrix, $A_{i j}$, the SVD method is obtained to solve the correlation matrix. This method provides a situation to apply POD even in non-square matrices. The SVD of an $m \times n$ matrix such as $A$, leads to a decomposition $A=U \times W \times V^{T}$, where $T$ stands for transpose, $U$ is an $m \times m$ orthogonal matrix that contains the left singular vectors; $V$ is $n \times n$ orthogonal matrix contains the right singular vectors. $W$ is an $m \times n$ positive definite matrix with diagonal entries contains $r$ nonzero elements, that known as singular values (eigenvalues) of matrix $A$, where $r$ is the rank of matrix $A$. $U$ and $V$ can be calculated as the eigenvectors associated with the nonzero eigenvalues of the (symmetric) matrices $A \cdot A^{T}$ and $A^{T} \cdot A$. Consequently, the SVD of $A$ is written as the following expression (Lathauwer et al., 2000):

$$
A_{i j}=\sum_{l=1}^{r} U_{i l} W_{l} V_{j l}^{T} .
$$

\subsection{Combined POD-SVD snapshot method with cubic spline interpolation}

Generally, in a POD-SVD solution, any set of data is decomposing into two parts, variables and constant data. The variables are determining an exclusive feature of each data set. In order to use this feature, the matrix of numerical data, at different angles of attack, for various Mach numbers, is considered as 
initial data. Instead of the angle of attack and Mach number, any other optional parameters also can be obtained. These parameters assumed to be arbitrary parameters such as $\delta$. A set of numerical or experimental data from flow field for different values of $\delta$ must be prepared, which is arranged intentionally. To predict the flow field for each value of $\delta$, which does not exist, an eigenvalue problem has to be solved. $\varphi^{k}$ is the eigenvalue of the flow field. To estimate the flow field for the desired values of $\delta$, the modal coefficients $\left(a^{k}\right)$ have to be interpolated or extrapolated. Hence, instead of interpolation between all grids of the field, combined POD-SVD snapshot method with an algorithm of interpolation or extrapolation is reconstructing the field just by obtaining an interpolation or extrapolation algorithm in modal coefficients $\left(a^{k}\right)$ (Najafbeygi et al., 2014). Through this method, the number of interpolation or extrapolation operations is going to reduce significantly. The most known and classic algorithm for interpolation is the cubic spline. Actually, this algorithm is based on a polynomial between two nodes. Splines produce a function that includes first and second derivatives. So, splines can be more stable than fitting some polynomial. In this case, the cubic spline will be continued among the different variations of $\left(a^{k}\right)$.

\section{High-order singular values decomposition}

HOSVD is a tensorial form of standard SVD, which only applies to matrices (Lorente et al., 2008). If $A$ is considered as a high-order tensor, it has been decomposed into the matrices. In this case, the snapshot tensor has been decomposed to the symmetric matrices to calculate the eigenvectors and eigenvalues. Consequently, it is possible to reconstruct a reduced order model of snapshot tensor A. Tensor decomposition is expressed by the following equation:

$$
A_{i j k}=\sum_{\eta}^{r_{1}} \sum_{\mu}^{r_{2}} \sum_{\zeta}^{r_{3}} \sigma_{\eta \mu \zeta} u_{i \eta} v_{j \mu} w_{k \zeta},
$$

where $\sigma_{\eta \mu \zeta}$ is the components of another third-order tensor, called the core tensor the (the order of the core tensor is always equivalent with the order of snapshot tensor). $u_{i \eta}, v_{j \mu}$ and $w_{k \zeta}$ are matrices that are known as HOSVD modes (eigenvectors) also $u_{i \eta}, v_{j \mu}, w_{k \zeta}$ are the components of some orthogonal systems. These are eigenvectors of symmetric matrices $B_{i l}^{1}$, $B_{j l}^{2}$ and $B_{k l}^{3}$. On the following expression, any tensor can be decomposed to the symmetric matrices. In this case, we applied a third-order tensor as the initial snapshot data. The first and second indexes of tensor $A_{i j k}$ are variations of the angle of attack and Mach number, and the third index is about the number of grids of the flow field. In fact, snapshot tensor can 
be in any order, and its order only depends on the number of various parameters. So, the HOSVD procedure is started by producing symmetric matrices as follow:

$$
\begin{aligned}
B_{i l}^{1} & =\sum_{j, k} A_{i j k} A_{l j k} \\
B_{j l}^{2} & =\sum_{i, k} A_{i j k} A_{i l k} \\
B_{k l}^{3} & =\sum_{i, j} A_{i j k} A_{i j l}
\end{aligned}
$$

The HOSVD modes are given by:

$$
\begin{gathered}
\sum_{l=1}^{m_{1}} B_{i l}^{1} u_{i \eta}=\left(\alpha_{\eta}\right)^{2} u_{i \eta}, \eta=1,2, \ldots, m_{1} \\
\sum_{l=1}^{m_{2}} B_{j l}^{2} v_{j \mu}=\left(\beta_{\mu}\right)^{2} v_{j \mu}, \mu=1,2, \ldots, m_{2} \\
\sum_{l=1}^{m_{3}} B_{k l}^{3} w_{k \zeta}=\left(\gamma_{\zeta}\right)^{2} w_{k \zeta}, \zeta=1,2, \ldots, m_{3},
\end{gathered}
$$

where, $m_{i}$ is the rank of the matrix $B^{i}$. The positive scalar amounts, $\alpha_{\eta}, \beta_{\mu}$ and $\gamma_{\zeta}$ are the high-order singular values of the snapshot tensor. When the calculation of the HOSVD modes done, the core tensor can calculate by the following equation:

$$
\sigma_{\eta \mu \zeta}=\sum_{i}^{m_{1}} \sum_{j}^{m_{2}} \sum_{k}^{m_{3}} A_{i j k} u_{i \eta} v_{j \mu} w_{k \zeta} .
$$

\subsection{Standard combined POD-HOSVD model}

To construct a reduced order model based on tensorial data, a similar manner with POD method has been used. HOSVD can decompose a tensor to the symmetric matrices (Equation (11)). Then, the POD approach is applied to compute the reduced order model of the field by eigenvalues and eigenvectors of these symmetric matrices (Equation (12)). The mentioned approach is creating a reduced order model, which is the combination of POD and HOSVD methods. The required number of modes to reconstruct an approximate model of the field is determined by the parameter $S_{i}$. This number can 

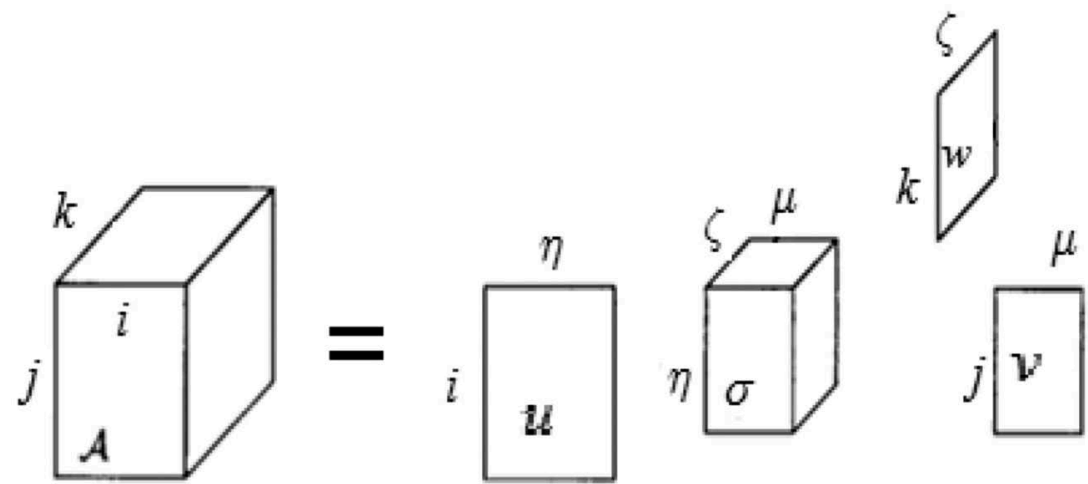

Figure 1. Standard tensor decomposition for a third-order snapshot tensor (Lathauwer et al., 2000).

be chosen by considering the relative energy of each mode. Consequently, the following expression is the reduced order model of tensor $A$ :

$$
A_{i j k} \cong \sum_{\eta}^{S_{1}} \sum_{\mu}^{S_{2}} \sum_{\zeta}^{S_{3}} \sigma_{\eta \mu} \zeta u_{i \eta} v_{j \mu} w_{k \zeta}
$$

where $S_{i} \leq m_{i}$. Figure 1 shows a schema of the standard tensor decomposition to a core tensor and three eigenvector matrices. At the following schema, the snapshot tensor decomposed to three plates which are the modes and a cube which is representing the core tensor (Lorente et al., 2008).

\section{Mode selection to construct a reduce order model}

Generally, in reduce order approaches, increasing the number of modes, leads to a more accurate model. Therefore, there is an optimum condition for choosing less number of modes to reconstruct the reduced order model (ROM) of the flow field. Thus, the total relative energy of modes is calculated, to choose the modes with the highest level of energy, by following equation (Bui-Thanh et al., 2004):

$$
k=\frac{\sum_{i=1}^{N_{r}} \lambda_{i}}{\sum_{i=1}^{N_{\text {Total }}} \lambda_{i}}
$$

where $\lambda$ is the eigenvalues of the snapshot matrix and $N_{\text {Total }}$ is the total number of snapshots for a certain parameter. If the value of $k$ is equal to 99.9\%, $N_{r}$ shows the optimum number of modes to reconstruct a reduced order model by sufficient accuracy. 
Algorithm 1: combined POD-SVD snapshot method with cubic spline interpolation

1. Prepare snapshots matrix

2. Calculate the eigenfunctions $\varphi^{k}$ and eigenvalues $\lambda$ of snapshot matrix by SVD algorithm

3. Estimate the eigenvalues of the field for the desired value of parameter $\delta$ by using a cubic spline interpolation algorithm on eigenvalues matrix.

4. Define order reduction criteria (Equation (15)) and choose the modes to construct a reduced order model.

5. Reconstruct the estimated field by modes that have been chosen in 4 and Equation (14).

\section{Cost efficient combined POD-HOSVD model}

In combined POD-HOSVD tensor decomposition procedure, the grids of the field always are defined as a variable parameter. In the standard combined POD-HOSVD method, $w_{k \zeta}$ is a symmetric matrix which is containing $m_{3} \times m_{3}$ arrays. The arrays of this matrix in each row and column are equal to the number of flow field grids. Hence, in the decomposition of snapshot tensor, computation of $w_{k \zeta}$ needs a lot of calculation time and storage capacity. Also, calculation of $\sigma_{\eta \mu \zeta}$ is another costly part of the HOSVD procedure. To define the required time for the reconstruction of the field, in addition to the $\sigma_{\eta \mu \zeta}$ and $w_{k \zeta}$ time consumption, we need to consider the required time for the inner product of these two variables. For instance, if the flow field in each snapshot has 9,600 grids, the number of arrays of $w_{k \zeta}$ will be around $9 \times 10^{7}$. So, production and involvement of $w_{k \zeta}$ in the calculation procedure is the beginning stage of computational difficulties. This problem conducted us to provide a cost-efficient combined PODHOSVD method. The following formulation is proposed, instead of Equation (13), to reduce the CPU time by the authors recently:

$$
\sigma_{\eta \mu k}=\sum_{i}^{m_{1}} \sum_{j}^{m_{2}} A_{i j k} u_{i \eta} v_{j \mu} .
$$

In Equation (16) $\sigma_{\eta \mu \zeta}$ changed to $\sigma_{\eta \mu k}$ and $w_{k \zeta}$ is eliminated (it can be demonstrated by continuum mechanics rules). $w_{k \zeta}$ is associated with properties of grids of the flow field. In the above formulation, the location 
properties of grids of one snapshot transferred to the core tensor and calculation of the matrix $w_{k \zeta}$ completely extracted from POD-HOSVD decomposition procedure. Finally, the reconstructed flow field is calculating, by the following equation:

$$
A_{i j k} \cong \sum_{\eta}^{S_{1}} \sum_{\mu}^{S_{2}} \sigma_{\eta \mu k} u_{i \eta} v_{j \mu} .
$$

Figure 2 shows a schema of cost-efficient tensorial decomposition. The core tensor $\sigma_{\eta \mu \mathrm{k}}$ is carrying the $k$ index. Therefore, it is possible to eliminate the matrix $w_{k \zeta}$.

Now with an interpolation or extrapolation algorithm, it is possible to estimate the eigenvectors of any desired field. So, Equation (17) can be written as the following expression:

$$
T_{k} \cong \sum_{\eta}^{S_{1}} \sum_{\mu}^{S_{2}} \sigma_{\eta \mu k} u_{i n t \eta} v_{i n t \mu},
$$

where $T_{k}$ is the matrix of desired filed which was not existing in the snapshot tensor. $u_{i n t \eta}$ and $v_{i n t \mu}$ are interpolated eigenvectors, and $\sigma_{\eta \mu k}$ is the transformed core tensor. In this case of study, $u$ and $v$ matrices are containing variations of Mach number and angles of attack respectively.

Algorithm 2 and 3 are the standard combined POD-HOSVD method and the cost-efficient combined POD-HOSVD method for the same snapshot tensor that includes several variables. The second, third and fourth steps of the standard combined POD-HOSVD are different from the cost-efficient combined POD-HOSVD method. The novelty of the presented method is on a new definition of the core tensor that is containing all grids of the field. Subsequently, it is no longer required
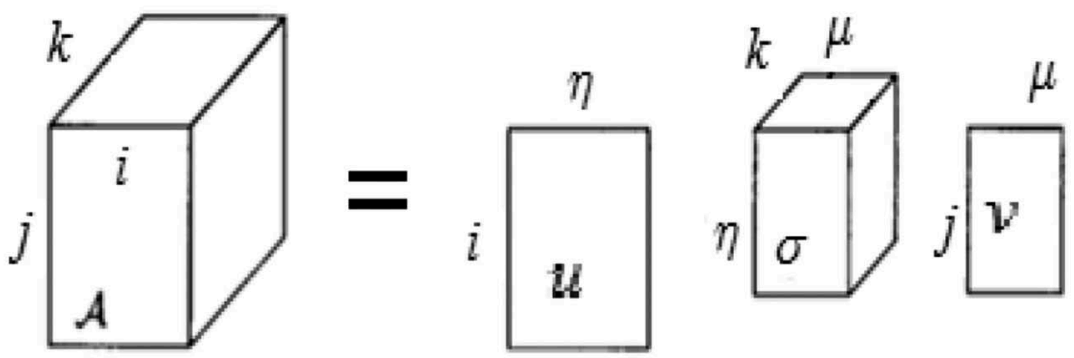

Figure 2. Schema of matrix elimination and index transfer, to reconstruct the reduce order model of tensor $A$. 
to compute the symmetric matrix of grids and calculating its eigenvectors to produce the core tensor.

Algorithm 2: Standard combined POD-HOSVD snapshot method with cubic spline interpolation

1. Preparing the snapshot tensor that includes several matrices $A_{1, \cdots, N}$.

2. Decomposition of any symmetric or asymmetric snapshots tensor to symmetric matrices by

$$
B_{i l}^{n}=\sum_{\forall n, n \neq l, i} A_{1, \cdots, i, \cdots, N} \cdot A_{1, \cdots, l, \cdots, N}
$$

$n$ is the number of parameters, $n=1, \ldots, N$

3. Calculation of the eigenvalues and eigenvectors of the symmetric matrices

$l$ is counting the variations of each parameter.

$n$ is the number of parameters.

$m_{n}$ is the total number of variations of each parameter.

$\alpha_{\eta}$ are the eigenvalues of the symmetric matrix.

$u_{i \eta}$ are the eigenvectors of the symmetric matrix.

$\sum_{l=1}^{m_{n}} B_{i l}^{n} u_{i \eta}=\left(\alpha_{\eta}\right)^{2} u_{i \eta}$,

$\eta=1,2, \ldots, m_{n}$

4. Calculation of the core tensor $\sigma_{1, \cdots, M}$ for the snapshots tensor $A_{1, \cdots, N}$. where $M$ is equal to $N$.

The core tensor is the result of the inner product of the snapshot tensor and symmetric matrices eigenvectors, which have been calculated at the third step.

5. Definition of the order reduction criterion (Equation (15)) and choosing the number of modes to construct the ROM.

6. Interpolation or extrapolation the eigenvectors to find the variables of the estimated field.

7. Constructing the estimated field as the result of the inner product of the core tensor and a sufficient number of eigenvectors for each parameter. 
Algorithm 3: Cost-efficient combined POD-SVD snapshot method by cubic spline interpolation

1. Preparing the snapshot tensor, contains several matrices $A_{1}, \ldots, N$.

2. Decomposing any symmetric or asymmetric snapshot tensor to symmetric matrices by

$$
B_{i l}^{n}=\sum_{\forall n, n \neq l, i} A_{1, \cdots, i, \cdots, N} \cdot A_{1, \cdots, l, \cdots, N}
$$

$n$ is the number of parameters. $n=1, \ldots, N-1$

There is no need to produce the symmetric matrix for grids.

3. Calculation of the eigenvalues and eigenvectors of the symmetric matrices by Lagrange algorithm.

4. Calculation of the core tensor by transferring the index of grids to the core tensor and eliminating the eigenvectors of grids from the core tensor calculation (Equation (16)).

5. Defining the order reduction criteria (Equation (15)) and choosing modes to construct the reduced order model.

6. Interpolating or extrapolating the eigenvectors to find the variables of the estimated field.

7. Constructing the estimated field as same as the standard method.

\section{CFD calculations of snapshots}

In this research, to obtain the preliminary data, which are mentioned above as snapshots, the CFD method has been used to calculate the aerodynamic coefficients of a NACA0012 airfoil in inviscid flow. The calculation was done by a $2.30 \mathrm{GHz}$ CPU, $8 \mathrm{~GB}$ RAM and 64-bit operating system. The Roe flux splitting method and circle (O Type) mesh with 9600 elements is considered for the CFD simulation. The CFL was assumed to be 1. Elements are generated as structured mesh, shown in Figure 3.

Initial data for variations of the angle of attack and Mach number in subsonic and supersonic flow are generated. Subsequently, snapshots can be divided into two main parts, subsonic and supersonic snapshots. To validate the initial data, in the following comparative example the numerical method has been compared with experimental results, in pressure coefficient of NACA0012 in Mach number of 0.8 and the angle of attack of 10 degrees in Reynolds number of 500. Figure 4 depicts the implementation of experimental results and CFD computation. 

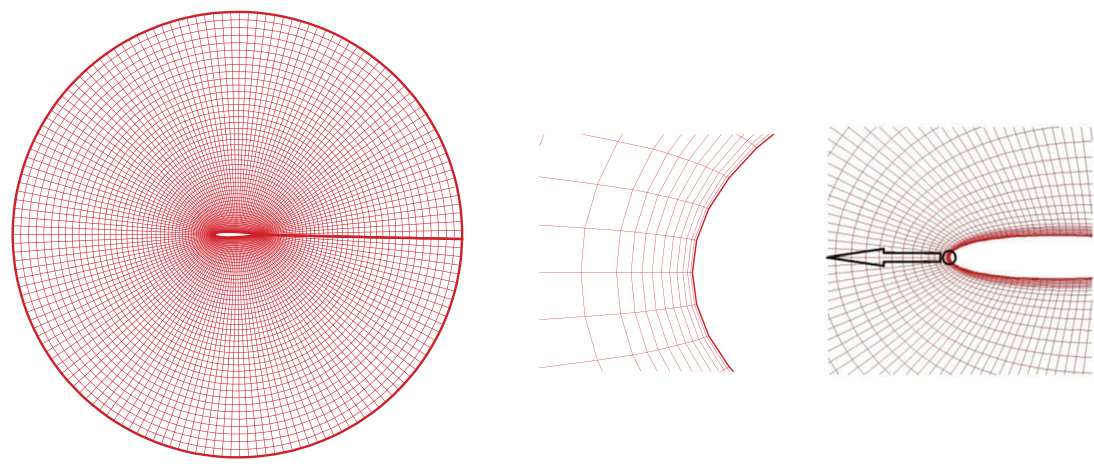

Figure 3. Structured mesh generation around the airfoil NACA0012.

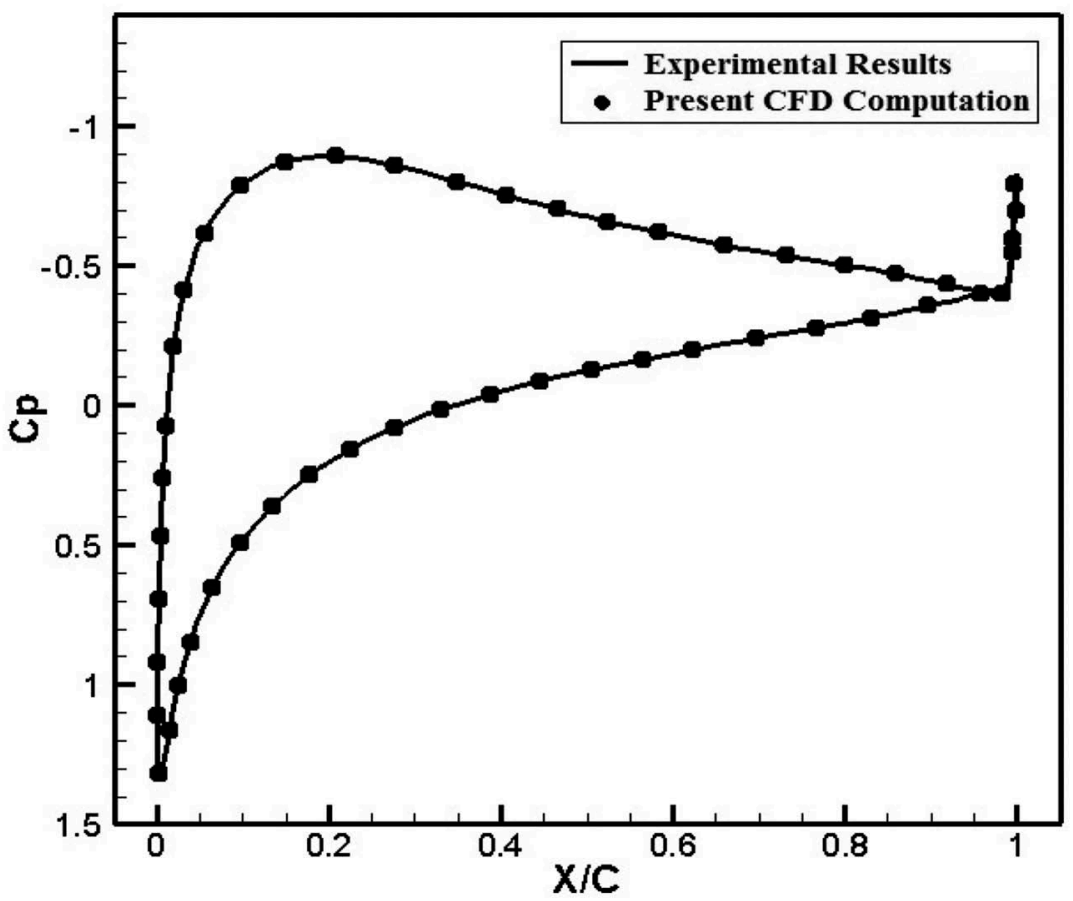

Figure 4. Pressure distribution on the surface of NACA0012 at $M_{\infty}=0.8$, Angle of attack $=10$ and $\operatorname{Re}=500$ (1993).

\section{Snapshots details}

Snapshots are divided into two parts such as subsonic and supersonic flow fields. In this study, the angle of attack and Mach number is as the variable parameters. There is no limitation on choosing the number of variable parameters. The angle of attack is changed between 2.5 and 17.5 degrees by step sizing of 2.5 degrees for both subsonic and supersonic flows. Mach number varies from 0.35 to 0.65 by 
the step sizing of 0.15 for subsonic flow. In the supersonic flow test case, Mach number variations are between 1.2 and 2.8 by the step size of 0.2 . In subsonic flow field, laminar compressible flow at the Reynolds number of 500 and in supersonic flow Reynolds number considered as 500,000.

\section{Results and discussion}

In general, snapshots included variables for subsonic and supersonic flows. In subsonic flow field, the variation of the angle of attack varies $2.5-17.5$ by 2.5-degree step size and the Mach number variation is $0.35-0.65$ by the step size of 0.15 and Reynolds number of 500. Also in supersonic flow variation of Mach number is between 1.2 and 2.8 with the step size of 0.2 . The angle of attack variance is the same as the subsonic flow field, and Reynolds number is 500,000. Combined POD-HOSVD and cost-efficient POD-HOSVD methods were compared in computational time consuming and required storage at the specified set of snapshots. To generate the missing data, the cost-efficient combined POD-HOSVD method was combined with the cubic spline interpolation method. Figure $5(\mathrm{a}, \mathrm{b})$ shows the variations of the relative energy of each mode

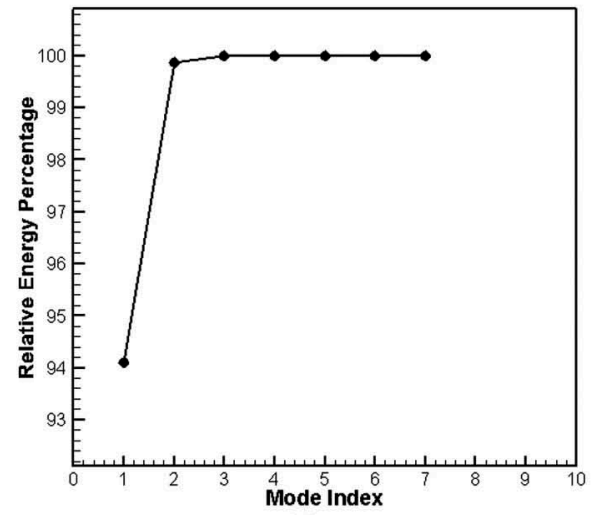

(a)

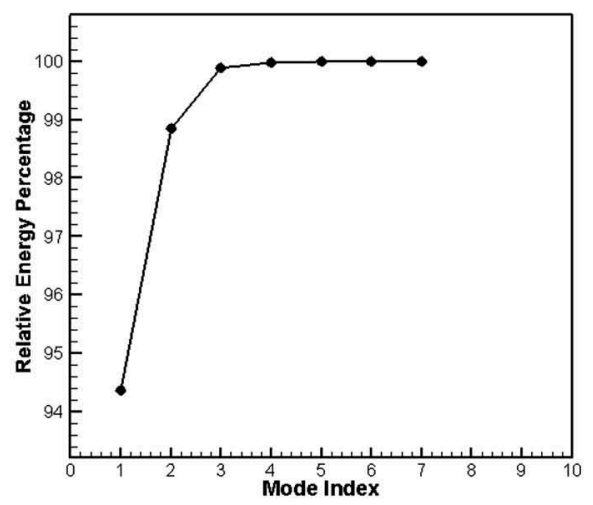

(c)

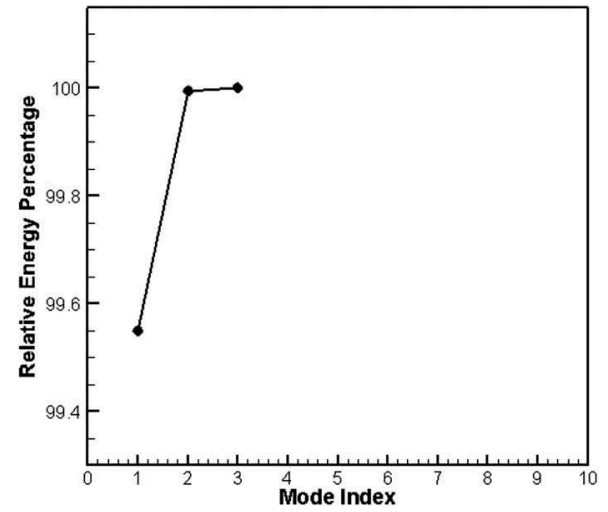

(b)

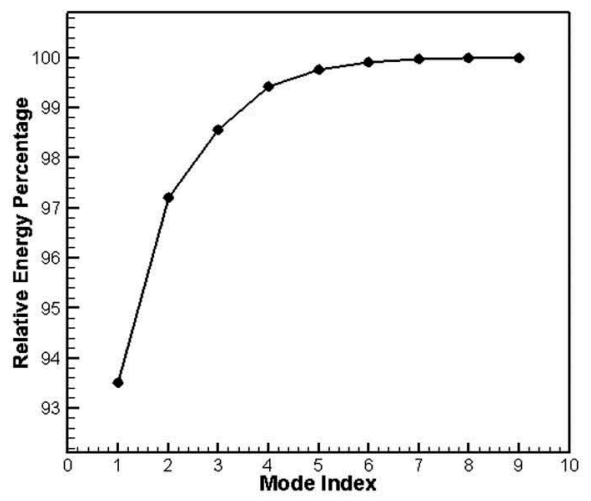

(d)

Figure 5. Relative energy spectrum of HOSDV modes: (a) angle of attack, (b) Mach number modes in subsonic flow filed, (c) angle of attack and (d) Mach number modes in supersonic flow filed. 
for the angle of attack and Mach number in subsonic flow. The total number of variation of Mach number and angle of attack in snapshot tensor is determining the number of generated modes. Hence, in subsonic flow filed seven and three modes for the angle of attack and Mach number respectively produced by decomposition. Calculation of the relative energy percentage of the angle of attack and Mach number shows that 99.9 percentage of relative energy is captured only by six modes of the angle of attack and two modes of Mach number. In the same figure, (c) and (d) depict the captured relative energy percentage by Mach number and angle of attack modes in supersonic flow. The

Table 1. Comparison of the computational time and storage capacity.

\begin{tabular}{lcccc}
\hline \multicolumn{1}{c}{ Method } & $\begin{array}{c}\text { Computational } \\
\text { time }(\mathrm{s})\end{array}$ & $\begin{array}{c}\text { Computational time percentage } \\
\text { in comparing with CFD }\end{array}$ & $\begin{array}{c}\text { Produced extra data } \\
\text { for calculation (MB) }\end{array}$ & $\begin{array}{c}\text { RAM } \\
\text { (MB) }\end{array}$ \\
\hline $\begin{array}{l}\text { CFD } \\
\text { Standard combined POD- } \\
\text { HOSVD }\end{array}$ & 550 & $100 \%$ & 7.2 & 160 \\
$\begin{array}{l}\text { Cost efficient combined } \\
\text { POD-HOSVD }\end{array}$ & 512 & $93.1 \%$ & 3,328 & 380 \\
\hline
\end{tabular}
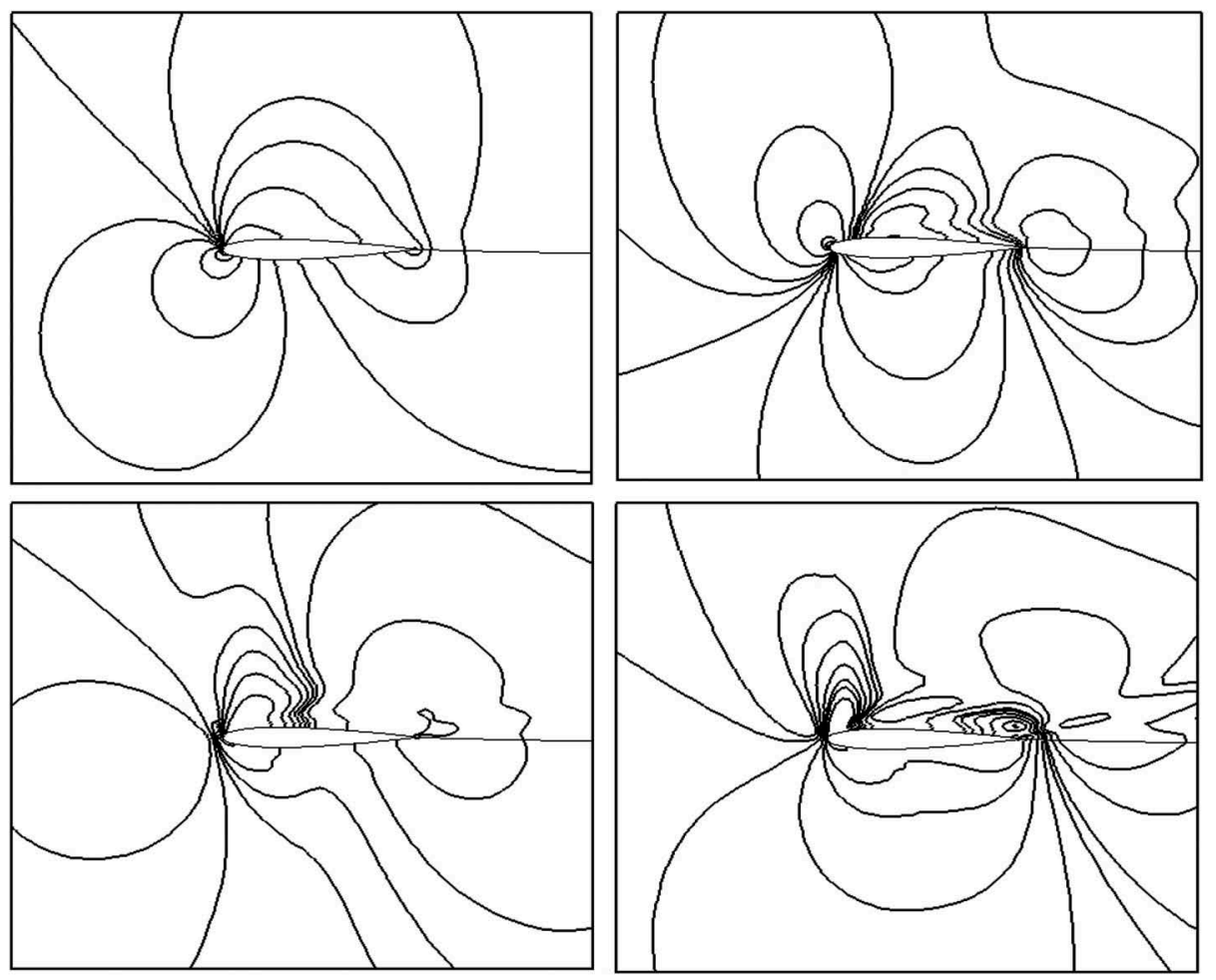

Figure 6. Contours of the first four strongest HOSVD modes of the angle of attack on the first mode of Mach number that captured the highest level of total kinetic energy of the subsonic flow field. 

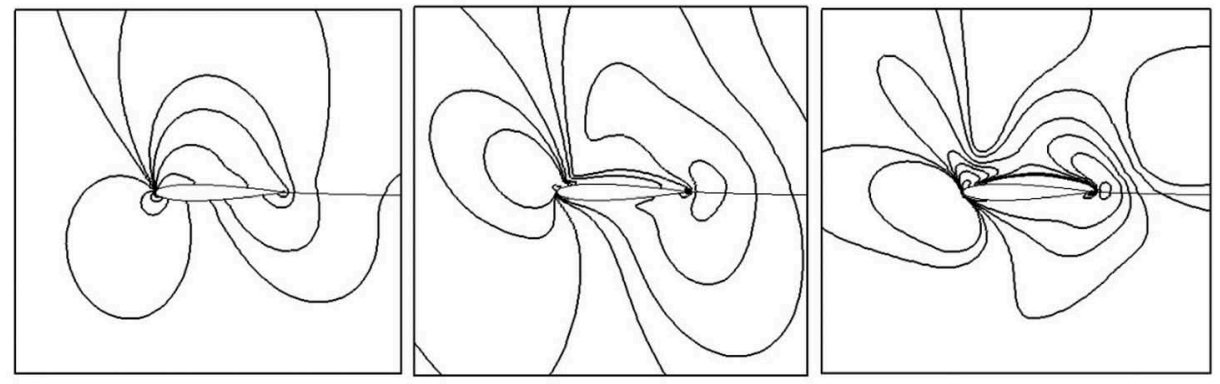

Figure 7. Contours of the first three strongest HOSVD modes of Mach number on the first mode of the angle of attack that captured the highest level of total kinetic energy of the subsonic flow field.
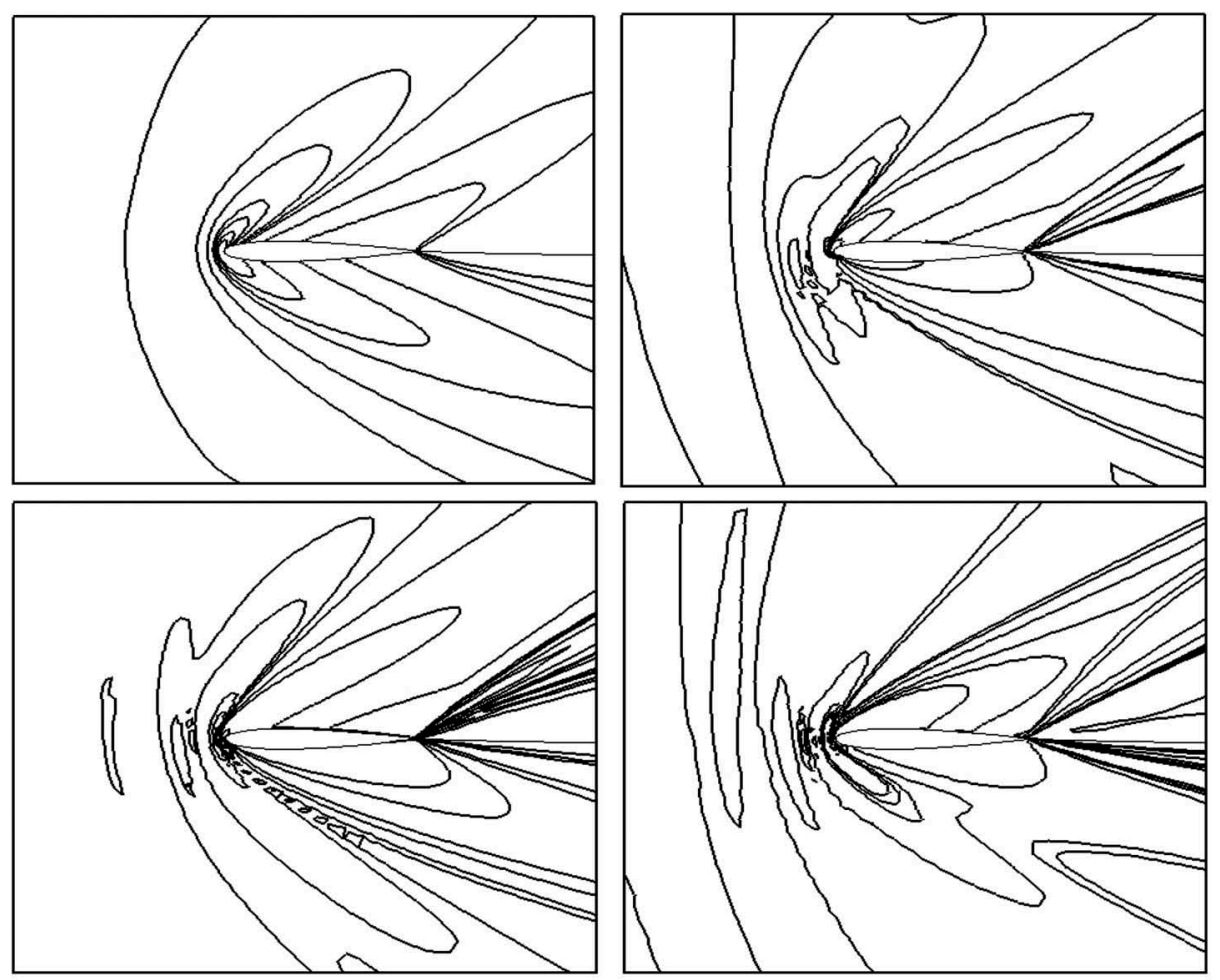

Figure 8. Contours of the first four strongest HOSVD modes of the angle of attack on the first mode of Mach number that captured the highest level of total kinetic energy of the supersonic flow field.

first five modes of the angle of attack and the first six modes of the Mach number are the most effective modes when they have captured the 99.9 percentage of relative energy.

Table 1 shows a comparison in computational time for subsonic flow field snapshot tensor between CFD and cost-efficient combined PODHOSVD methods. This tensor has 201,600 arrays. The cost-efficient 

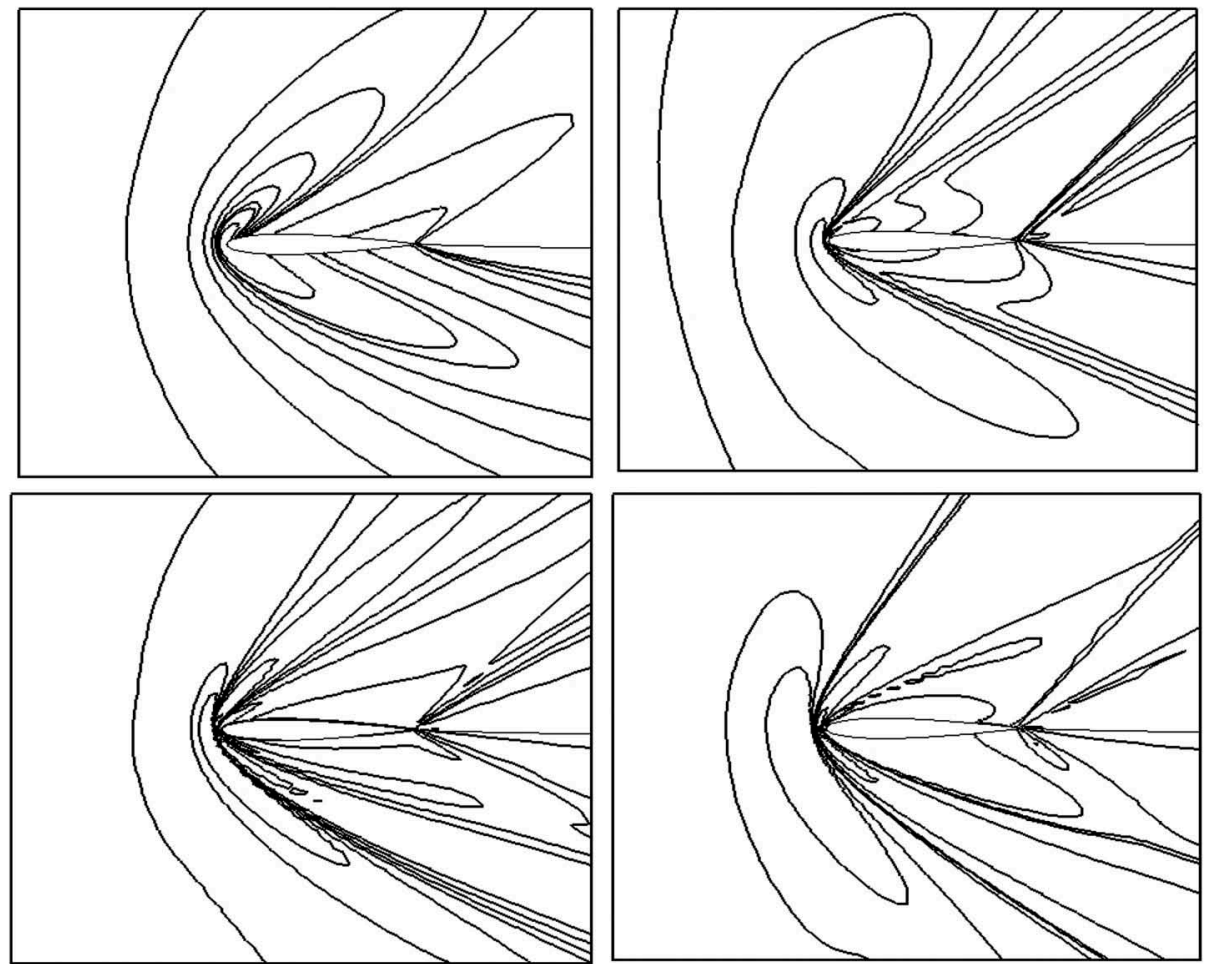

Figure 9. Contours of the first four strongest HOSVD modes of Mach number on the first mode of the angle of attack that captured the highest level of total kinetic energy of the subsonic flow field.

combined POD-HOSVD approach is around 100 times faster than regular combined POD-HOSVD method. Results indicate that standard combined POD-HOSVD approach produces around 3 GB more data.

The subsonic flow was modelled only by six modes of the angle of attack and two modes of Mach number. Also, in supersonic modelling five modes of the angle of attack and six modes of Mach number have been used. Figure 6 shows HOSVD modal distribution in subsonic flow for the first four modes of the angle of attack on the first mode of Mach number. Figure 7 depicts the modal distribution of the first three modes of Mach number in the first mode of the angle of attack.

By Figures 6 and 7, it is evident that the first mode captured a higher level of the kinetic energy of the field. So, it is the most detailed modes of the field. Figure 8 shows mode distribution in supersonic flow field for the first four modes of the angle of attack and the first mode of Mach number. Figure 9 is the HOSVD modes for the first four modes of Mach number and first mode of the angle of attack.

Generally, despite the longer distance of angle of attack variations (in comparison with Mach number variance in subsonic and supersonic flow fields) the modes of the angle of attack captured a higher level of relative energy. 


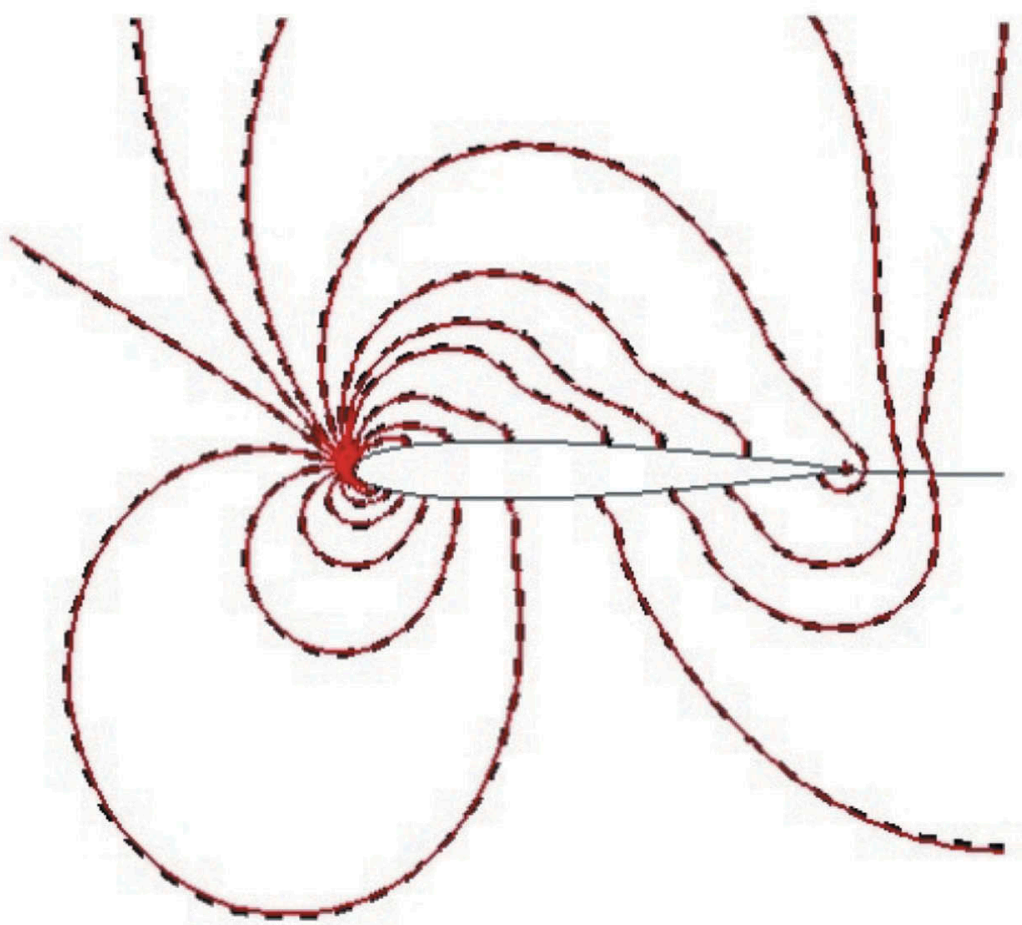

Figure 10. Contour of the pressure coefficient in 13 degrees and the Mach number of 0.45 , solid lines are CFD data, dashed lines are reconstructed filed using CE-POD-HOSVD model.

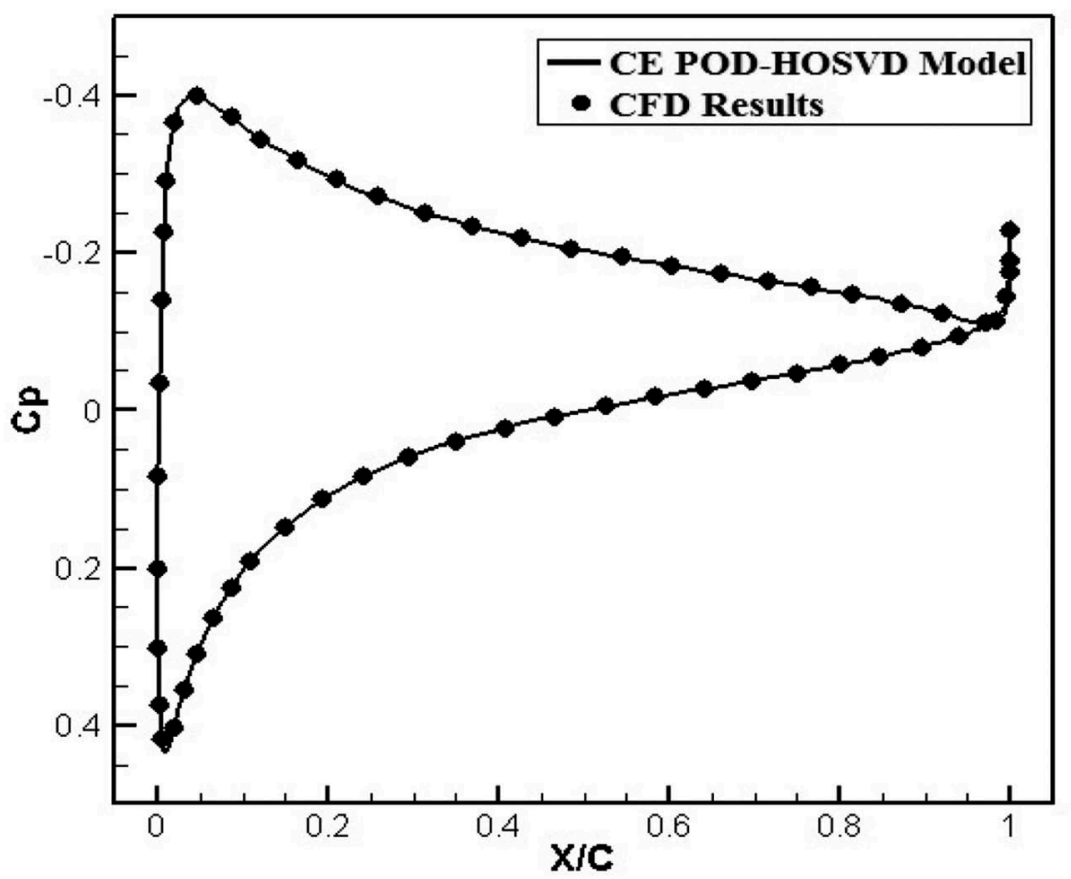

Figure 11. Surface pressure distribution in 13 degrees and the Mach number of 0.45 . 


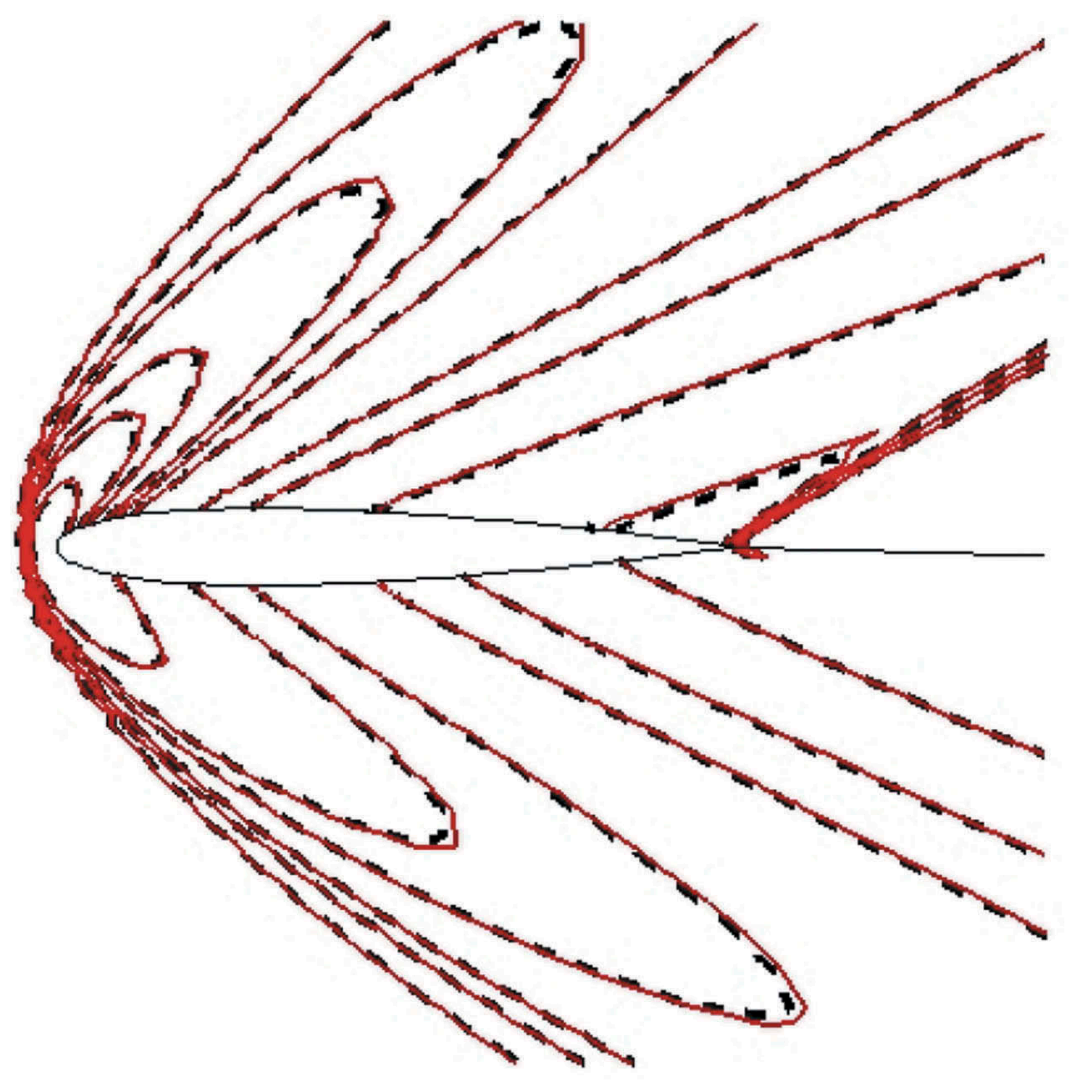

Figure 12. Contour of the pressure coefficient at an angle of attack of 8 degrees and the Mach number of 2.1, solid lines are CFD data, dashed lines are reconstructed filed using CE-POD-HOSVD model.

It can be concluded that this approach is more sensitive to Mach number variance than the angle of attack.

According to the results of the surface pressure coefficient distribution of airfoil NACA0012 and pressure distribution of the flow field, it can be obtained, that in subsonic and supersonic flows, the reconstructed field, highly matched with the numerical data especially in the near distances to the surface of the airfoil (Figure 10-13). Therefore, constructed data can be applied to estimate the airfoil drag and lift. Table 2 shows the comparison of the lift and drag coefficients in subsonic and supersonic flows between CFD results and cost-efficient combined POD-HOSVD method.

Results indicate that cost-efficient combined POD-HOSVD approach is a highly reliable method. Thus, the skin friction coefficient is one of the most sensitive data of an airfoil in the flow field. Results directly refer to the accuracy of approach in the near distance of the airfoil in subsonic and supersonic flows. According to the obtained data, cost-efficient combined POD-HOSVD can be 


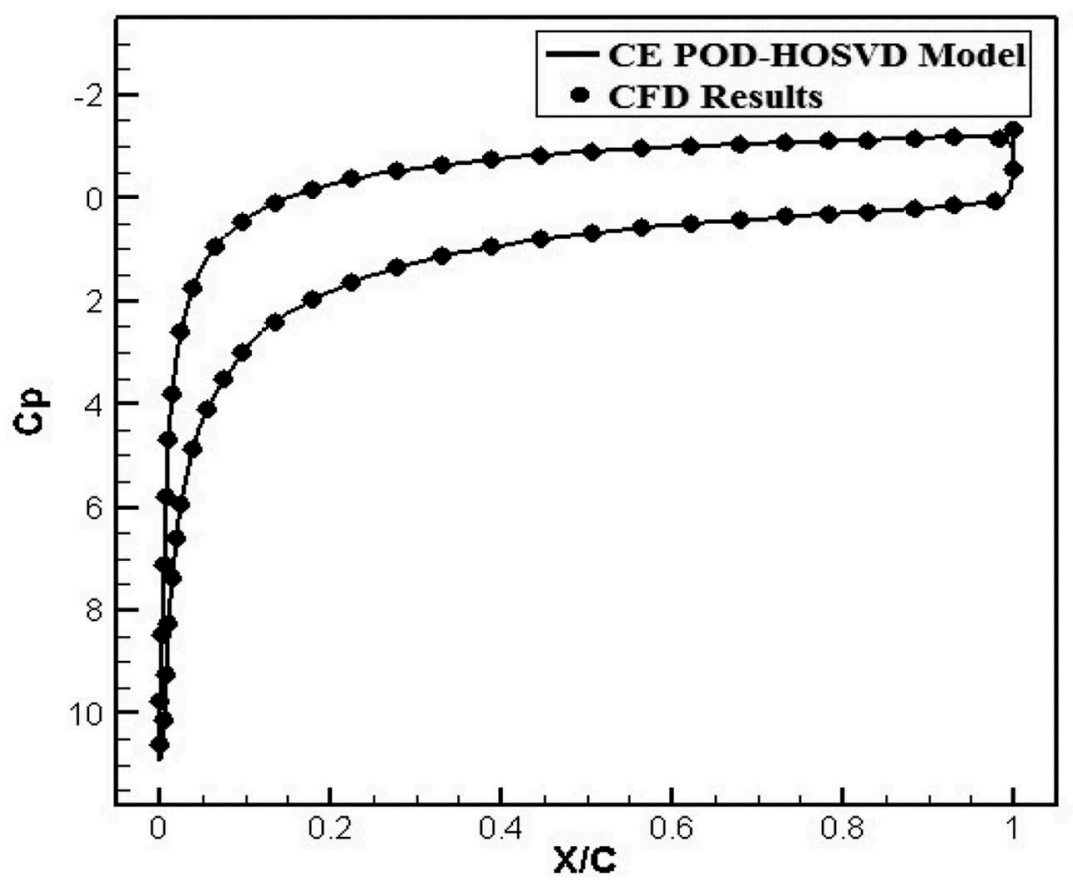

Figure 13. Surface pressure distribution at an angle of attack 8 degrees and the Mach number of 2.1.

Table 2. Comparison of the lift and drag coefficients.

\begin{tabular}{llcc}
\hline & \multicolumn{1}{c}{ CFD } & $\begin{array}{c}\text { Cost efficient combined } \\
\text { POD-HOSVD }\end{array}$ & $\begin{array}{c}\text { Error in comparing } \\
\text { with CFD }\end{array}$ \\
\hline Lift coefficient (subsonic flow) & 0.4096171 & 0.4119045 & $0.56 \%$ \\
Drag coefficient (subsonic flow) & $6.4353794 \mathrm{E}-02$ & $6.4585261 \mathrm{E}-02$ & $0.36 \%$ \\
Lift coefficient (supersonic flow) & 0.8405706 & 0.8324854 & $0.96 \%$ \\
Drag coefficient (supersonic flow) & $8.1440508 \mathrm{E}-03$ & $7.8240037 \mathrm{E}-03$ & $3.93 \%$ \\
\hline
\end{tabular}

applied to different parameters of the field (see Figure 14-15). Also, the aerodynamic coefficients data acknowledge a very high accuracy of the method in flow field estimation. Relative error percentage contour of each element in subsonic and supersonic fields show the deviation of the constructed field with CFD simulation for each element. It is observed that in subsonic flow, most parts of the field matched precisely with CFD results (Figure 16). In supersonic flow, only after the shock wave, it can estimate properly (Figure 17). Also, in supersonic flow, it can be a reliable scheme for flow field after the shock wave, nearby the airfoil. Supersonic flows can be categorised in the hyperbolic problems which are two primary characteristics lines. Due to this reason and the creation of shock waves in the flow field, the transmission of information in the field is performed in two regions which are called active and 


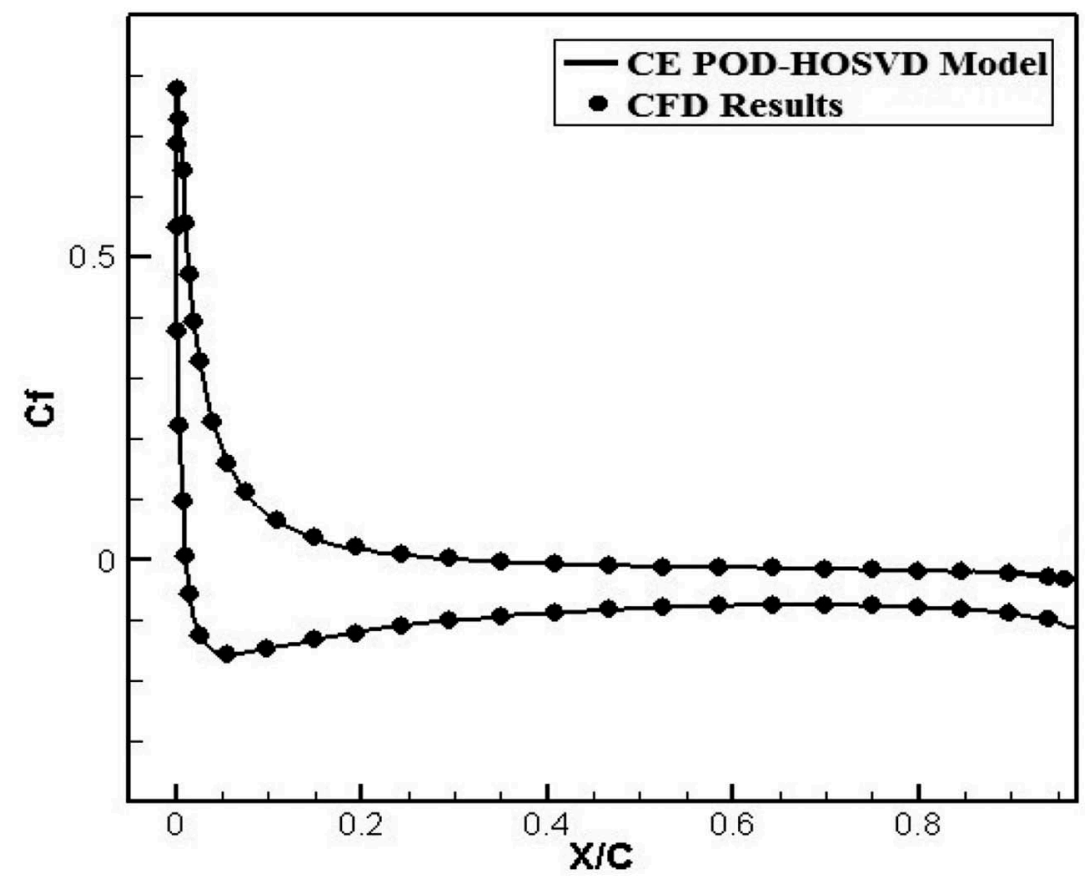

Figure 14. Skin friction coefficient distribution at an angle of attack 13 degrees and the Mach number of 0.45 .

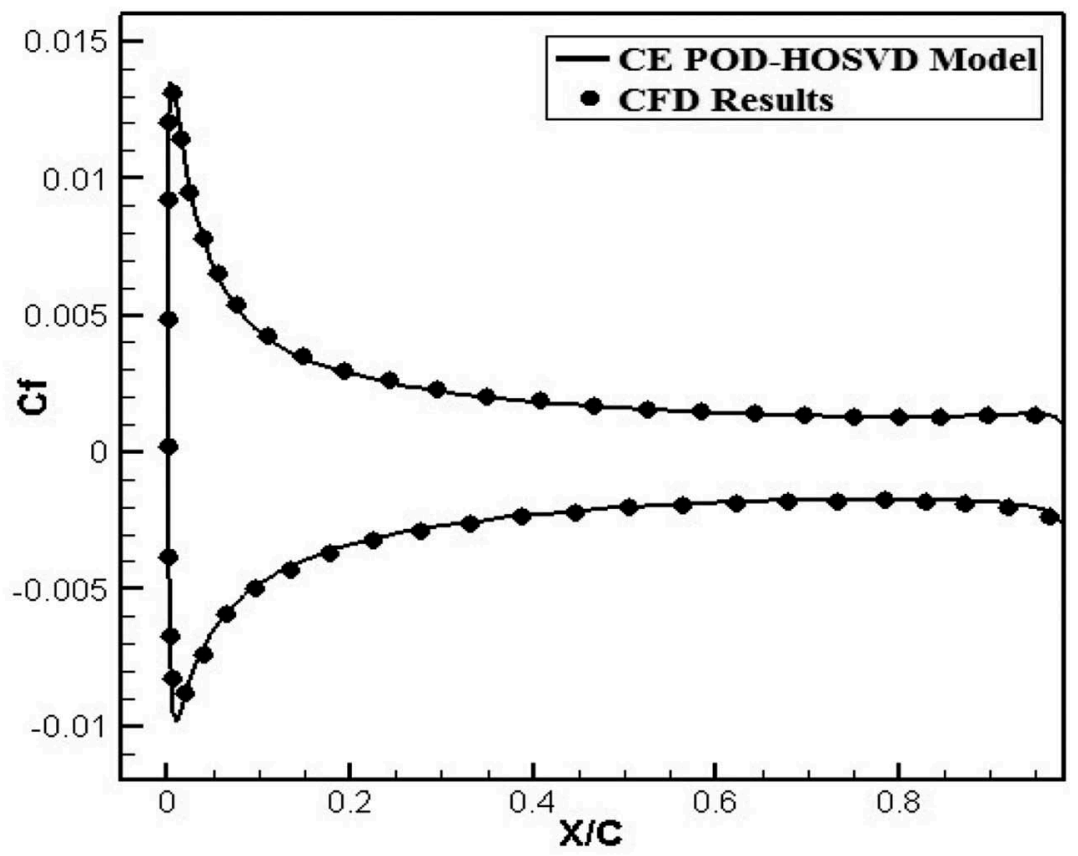

Figure 15. Skin friction coefficient distribution at an angle of attack of 8 degrees and the Mach number of 2.1. 


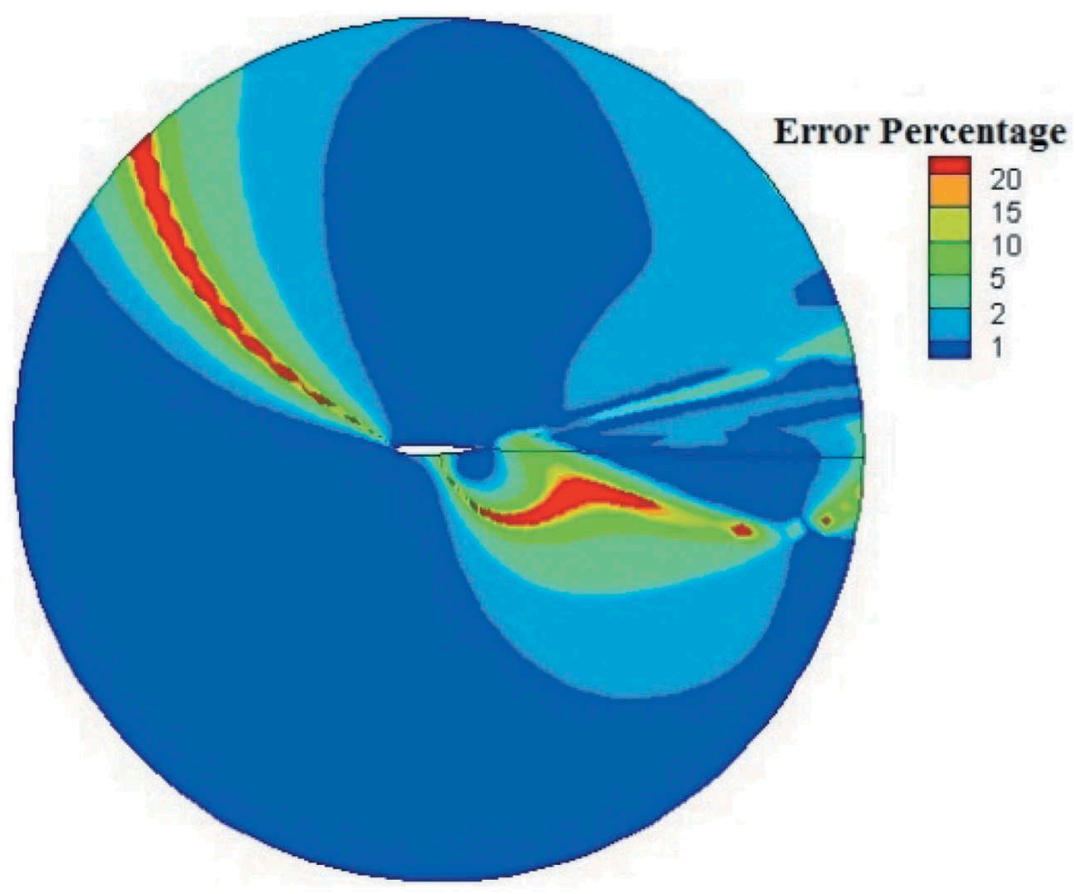

Figure 16. Contour of the relative error of pressure coefficient in subsonic flow field at an angle of attack of 13 degrees and the Mach number of 0.45 .

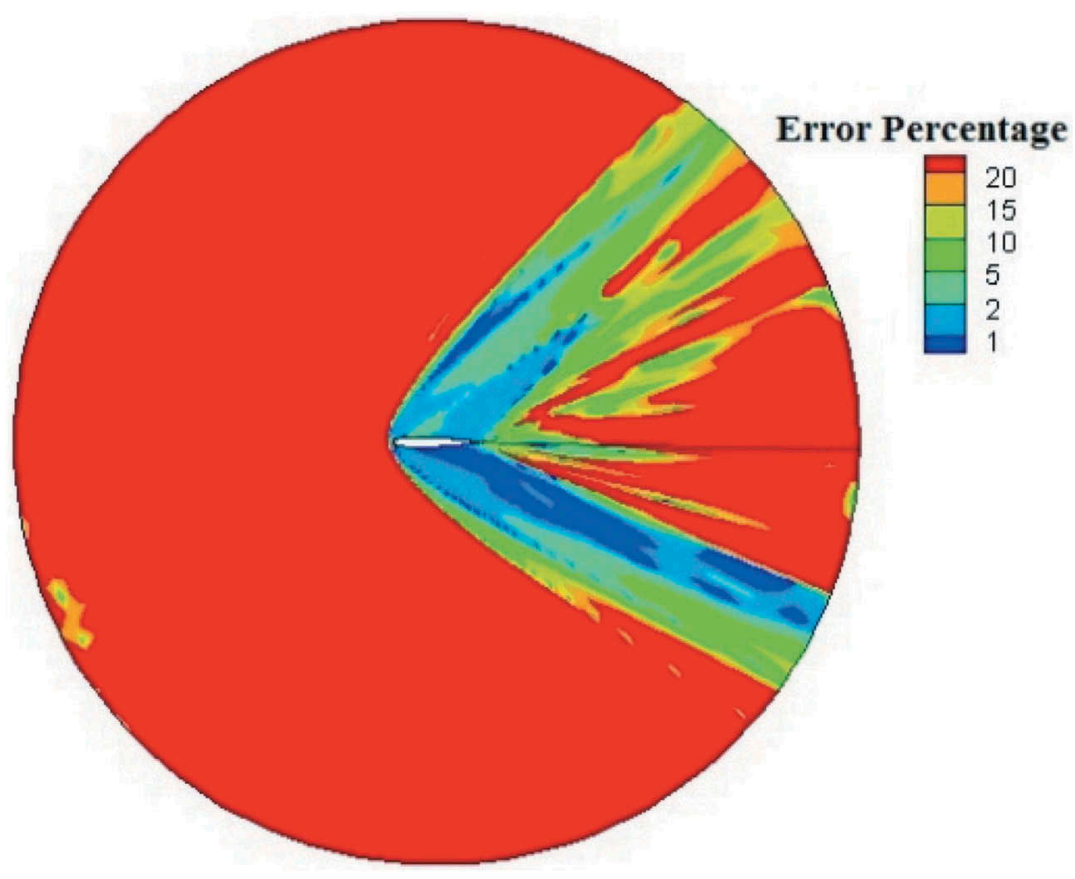

Figure 17. Contour of the relative error of pressure coefficient in supersonic flow field at an angle of attack of 8 degrees and the Mach number of 2.1. 
silent. With respect to the reasons which are mentioned above, the flow field in the downstream of the shock wave is more important for the prediction of aerodynamic data. One of the numerical approaches for the solution of supersonic flows is a shock fitting scheme. In this scheme, the flow field in the downstream of the shock wave is more important. Therefore, the higher error of the flow field in the upstream region is not very important for the calculation of aerodynamic data in the supersonic flow regime.

\section{Conclusion}

Standard combined POD-HOSVD is a powerful method to reconstruct the field with various numbers of effective parameters. Besides this advantage, the HOSVD approach has a lack in the reconstruction of the whole flow field with a large number of elements (grids). The challenge was overcome by the formulation which was presented in this research. HOSVD method was formulated independently from the spatial grids of snapshots. So, the elimination of a huge matrix from calculation indicates that the cost-efficient combined POD-HOSVD method is significantly faster than regular methods. Based on the results, for a specific test case, the cost-efficient combined POD-HOSVD approach is about 100 times faster than standard combined POD-HOSVD method. That makes it a good choice for reconstruction of the missing or incomplete data, without time-consuming of CFD simulation. Also, the costefficient combined POD-HOSVD method can be applied as a fast preprocessing approach. The error percentage of the estimated flow field in subsonic and supersonic flows shows that the approach has sufficient accuracy to estimate integral data such as lift and drag coefficients and the simulation of the whole flow field. The proposed model can be applied for any problems with a snapshots tensor under the variations of some effective parameters such as modelling of a control system, inverse design and inverse problems, and free vibration of beam models under the effect of different loadings and changes of the effective parameters.

\section{Disclosure statement}

No potential conflict of interest was reported by the authors.

\section{References}

Bergmann, M., \& Cordier, L. (2008). Optimal control of the cylinder wake in the laminar regime by trust-region methods and POD reduced order models. Journal of Computational Physics, 227(16), 7813-7840.

Berkant, S., \& Lars, E. (2006, August). Handwritten digit classification using higher-order singular value decomposition. Journal of Pattern Recognition, 40(3), 993-1003. 
Bourguet, R., Braza, M., \& Dervieux, A. (2011). Reduced-order modeling of transonic flows around an airfoil submitted to small deformations. Journal of Computational Physics, 230, 159-184.

Bui-Thanh, T., Damodaran, M., \& Willcox, K. E. (2004, August). Aerodynamic data reconstruction and inverse design using proper orthogonal decomposition. AIAA Journal, 42 1505-1516

Chambes, D. H., Adrian, R. J., Moin, P., Stewart, D. S., \& Sung, H. J. (1988). KarhunenLoeve expansion of Burger's model of turbulence. Physics of Fluids, 31, 2573-2582.

Connell, R. J., Kulasiri, D., Lennon, J., \& Hill, D. F. (2007). Computational modeling of turbulent velocity structures for an open channel flow using karhunen-loéve expansion. International Journal of Computational Methods, 4(3), 493-519. doi:10.1142/ S0219876207001242

Dowell, E., Hall, K., Thomas, J., Florea, R., Epureanu, B., \& Hegg, J. (1999). Reduced order models in unsteady aerodynamics (AIAA Paper 99-1261).

Dutta, V. (1993). An implicit finite volume nodal point scheme for the solution of two-dimensional compressible Navier-Stokes equations. Bangalore, India: National Aerospace Laboratories.

Holmes, P., Lumley, J. L., \& Berkooz, G. (1996). Turbulence coherent structures, dynamical systems and symmetry. Cambridge, UK: Cambridge University Press.

Karhunen, K. (1946). Zur spektraltheorie stochastischer prozesse. Annales Academiae Scientiarum Fennicae, Series A. 1, Mathematica-physica.

Lathauwer, L. D., Moor, B. D., \& Vandewalle, J. (2000, April). A multilinear singular value decomposition. SIAM Journal on Matrix Analysis and Applications, 21(4), 1253-1278.

Leschziner, M. A., Fishpool, G. M., \& Lardeau, S. (2009). Turbulent shear flow: A paradigmatic multiscale phenomenon. Journal of Multiscale Modelling, 1(2), 197-222.

Lieu, T., \& Farhat, C. (2005, December). Adaptation of POD-based aeroelastic ROMs for varying Mach number and angle of attack: Application to a complete F16 configuration. U. S. Air Force T\&E Days, U.S. Air Force T\&E Days Conferences.

Lorente, L. S., Vega, J. M., \& Velazquez, A. (2008, September-October). Generation of aerodynamic databases using high-order singular value decomposition. Journal of Aircraft, 45(5), 1779-1788.

Lucia, D. J. (2001). Reduce order modeling for high speed flows with moving shocks (Ph.D. Thesis). Department of the Air Force, Air university.

Lumley, J. L. (1967). The structure of inhomogeneous turbulence. In A. M. Yaglom \& V. I. Tatarski (Eds.), Atmospheric turbulence and wave propagation (pp. 166-178). Nauka, Moscow.

Martin, H., Roemer, F., \& Del Galdo, G. (2008). Higher-order SVD-based subspace estimation to improve the parameter estimation accuracy in multidimensional harmonic retrieval problems. IEEE Transactions on Signal Processing, 56(7), 3198-3213. doi:10.1109/TSP.2008.917929

Moayyedi, M. K., Najafi, M. R., \& Najafbeygi, M. (2014, December). A low-dimensional POD-HOSVD model for free vibration simulation of linear beam under variations of several parameters. The 4th Conference on Acoustics and Vibration. Tehran: Iran University of Science and Technology.

Moayyedi, M. K., Sabour, M. H., Najafbeygi, M., \& Hojaji, M. (2013). Development of a reduced-order model for flow field estimation based on the proper orthogonal decomposition. FD2013 Conference. Hormozghan University.

Najafbeygi, M., Sabour, M. H., Moayyedi, M. K., \& Hojaji, M. (2014, March). Flow field estimation due to several parameters variations based on combined POD/HOSVD reduced-order model, 13th Conference of Iranian Aerospace Society. University of Tehran. 
Romanousk, M. (1996). Reduced order unsteady aerodynamics and aeroelastic models using Karhunen-Loeve eigenmodes (AIAA, Paper 96-3981).

Sharma, A. S. (2009). Model reduction of turbulent fluid flows using the supply rate. International Journal of Bifurcation and Chaos, 19(4), 1267-1278.

Sirovich, L., \& Kirby, M. (1987). Low-dimensional procedure for the characterization of human faces. Journal of the Optical Society of America, 4(3), 519-524.

Ştefănescu, R., Sandu, A., \& Navon, I. M. (2014). Comparison of POD reduced order strategies for the nonlinear $2 \mathrm{D}$ shallow water equations. International Journal for Numerical Methods in Fluids, 76, 497-521. 